\title{
Implementation of FMAC in Healthcare System of Wireless Sensor Network
}

\author{
Ms. Poonam Shelar, PG Scholar, University of Mumbai, India poonamshelar313@yahoo.in \\ Prof. Uttara Gogate, Associate Professor, Shivajirao S. Jondhale College of Engineering, \\ Mumbai, India uttaragogate@yahoo.co.in
}

\begin{abstract}
Wireless sensor network is the network of sensor nodes. The nodes transmit data to the sink node. Each sensor nodes have its own battery and ability to communicate with sink node. Network of such nodes is called sensor network. The successful operation of WSN largely depends on MAC sublayer which deals with addressing and channel access control. WSN has varied design constraints such as energy consumption, scalability, delay, traffic control, packet delivery throughput and overheads that need an effective MAC protocol to deal with these problems. Energy must be utilized efficiently in order to increase the lifetime of the Wireless Sensor Network. MAC protocols reduce the cost of energy consumption by providing an efficient communication for transmission and hence improves network lifetime. This paper shows the implementation of FMAC Protocol in healthcare system for different reporting rates. The purpose of the study is to identify the best MAC protocol in Healthcare System. Results for implementation of Hybrid MAC for hospital network are presented in the paper. Results for Data transmission between nodes and sink node has been evaluated to identify Good MAC protocol. Paper represents how low energy consumption can be achieved by combining TDMA and CSMA MAC protocols.
\end{abstract}

Keywords - Wireless sensor network, Healthcare System, Sink node, Carrier sense multiple access (CSMA), Time division multiple access (TDMA), Federated MAC (FMAC).

\section{INTRODUCTION}

Wireless sensor networking is an emerging technology which provides a wide range of potential applications including environment monitoring, smart spaces, medical systems and robotic exploration. In wireless sensor networks source node use to send data packets to the destination (Sink) node. In such a network a large number of distributed nodes are present that organize themselves into a multi-hop wireless network. One or more sensors are present in each node, embedded processors and low-power radios, and is normally battery operated. Typically, these all nodes coordinate and communicate each other to perform a common task. Same as other shared-medium networks, medium access control (MAC)is an important technique in WSN that enables the successful operation of the network. Performance of the net- work is depending upon the congestion and collision of the packets near the sink node. Collision avoidance is one of the fundamental tasks of the MAC protocol so that two interfering nodes do not transmit at the same time. There are several MAC protocols that have been developed for wireless voice and data communication networks. CSMA, TDMA, 802.15.4, SMAC are the MAC Protocols of the wireless sensor networks. TDMA is working with slot time allocation mechanism. Performance of the network is depending upon the congestion and collision of the packets near the sink node. CSMA, TDMA, 802.15.4, SMAC are the MAC Protocols of the wireless sensor networks. TDMA is working with slot time allocation mechanism, where each node gets timeslot for transferring data to the sink node. Performance of the network should be increases for energy consumption and throughput with increasing number of slots. But nodes implementing TDMA needs to wait till it get timeslot. Due to this delay can be introduce and throughput may get decrease [1]. Different kinds of technique can be used for data collection. Centralize data collection technique minimizes the end to end delay and energy consumption [2]. In situation of congestion and heavy traffic load increases in the network. Then the alternative path for data transmission should be used. Alternative paths help for reduce heavy traffic load and congestion in the network [3]. Congestion and collision is the major issue in the wireless sensor networks. Congestion will occur due to the heavy traffic and hidden nodes problem. Due to the hidden node collision should be occurs in the network. Maximum number of packets lost due to the collision. Dropped packets sent using retransmission. So, Queen MAC solve the problem of hidden nodes [4]. For communication purpose mobile sink or static sink should be used. Mobile sink is very good for data collection process in sensor network. But static sink gives better performance 
for more quality of services of wireless sensor networks [5]. CSMA- based MAC protocol for wireless sensor networks to be able to save the power consumption, manage the mobility, self- organization, and failure recovery strategies. Both schedules based and CSMA based MAC protocols using in wireless sensor network have advantages and drawbacks. It depends on applications we can choose the suitable protocols. The combination of these two protocols is an efficient way of implementing special MAC protocol for WSN, namely hybrid protocols [6]. By applying WSN technology in the field of industrial monitoring or healthcare monitoring the single CSMA or TDMA protocol cannot meet the requirement of dealing with the urgent data without delay when some accidents occur. In order to solve this problem, author puts forward a hybrid CSMA-TDMA MAC protocol for wireless sensor network, and makes a test in a star network which is made up of one centre node and several sensor nodes [7]. In [8] author has evaluated performance of FMAC for varying the size of packet. In their proposed scenario, packet size is varied from 50 bytes to 250 bytes. The proposed protocol gave better performance for Average packet delivery ratio than TDMA, CSMA and 802.15.4. Also, Average End-to-End Delay less as compare to CSMA and 802.15.4. Average Throughput for FMAC is better as compare to TDMA, 802.15.4 and CSMA. But Average energy consumption is $25 \%$ more for FMAC as compare to TDMA and less as compare to 802.15.4 and CSMA. Packet loss ratio of FMAC was very less as compare to 802.15.4 [8]. For practical application of WSN, limited energy source is the major problem along with this problem transmission performance, throughput must be improved too. To tackle both of the issues simultaneously, author has proposed a hybrid TDMA/CSMA MAC layer protocol, by taking the advantages of Time Division Multiple Access (TDMA) and Carrier Sense Multiple Access (CSMA) at the medium access control (MAC) layer of WSN [9].

\section{PROPOSED SYSTEM}

In the proposed system scenario of healthcare system is considered where we have deployed 30 wireless sensor nodes. In the same network we classified 30 nodes in two types, among 30 nodes 4 nodes are working as Prior nodes, one is sink node and remaining are normal nodes. In this network excluding prior nodes all remaining nodes works with existing protocol TDMA MAC protocol. In Timedivision multiple access (TDMA) wireless sensor nodes transmit the packets in rapid succession, one after the other, each using its own time slot. But in Hospital network in case of an emergency situation data communication from sensor network must be done with minimum delay. For handling such situation Prior nodes are designed which accesses the channel using CSMA protocol. Here In the proposed method, the communication during the Data Exchange Period can be divided into two parts: the first part is communication in CSMA and the second is in TDMA.
Prior nodes have higher priority to communicate in CSMA, while normal nodes in TDMA. After receiving all the data of prior nodes, the centre node will check the address table, knowing what the other nodes in address table which have not sent data yet are normal nodes. Then the centre node will send synchronization command involving the information of time slot assignment in address order for all normal nodes. Each normal node can pick up its own slot priority from the synchronous command, and then sets exact timing so as to send data in its own slot. During the timing, the sensor falls into a doze to save energy. In addition, if the centre node does not receive any data from a sensor node in three continuous cycles, the sensor node will be considered out of network owing to power exhausted, and its record in the address table will be deleted, meanwhile a sound and light alarm will be given by centre node.

\section{A. Simulation model for System}

\begin{tabular}{|c|c|}
\hline SIMULATOR & Network Simulator 2.35 \\
\hline NUMBER OF NODES & 30 \\
\hline AREA & $600 \mathrm{~m}$ x 600m \\
\hline COMMUNICATION RANGE & $250 \mathrm{~m}$ \\
\hline PACKET SIZE & 512 bytes \\
\hline INTERFACE TYPE & Phy/WirelessPhy \\
\hline MAC TYPE & IEEE 802.11, TDMA, FMAC, \\
\hline QUEUE TYPE & DropTail/Priority Queue \\
\hline QUEUE LENGTH & Omni Antenna \\
\hline ANTENNA TYPE & TwoRayGround \\
\hline PROPAGATION TYPE & AODV \\
\hline ROUTING PROTOCOL & UDP \\
\hline TRANSPORT AGENT & CBR \\
\hline APPLICATION AGENT & 50 Seconds \\
\hline SIMULATION TIME & Thodel \\
\hline
\end{tabular}

Table 1. Simulation model

\section{B. Network Architecture}

Node (0) is sink node, Node (1) is patient ward monitoring node, Node (2) is ICU monitoring node, Node (3) is casualty node, Node (4) is OP node. Node 1,2,3,4 are high priority nodes. All other nodes are normal nodes.

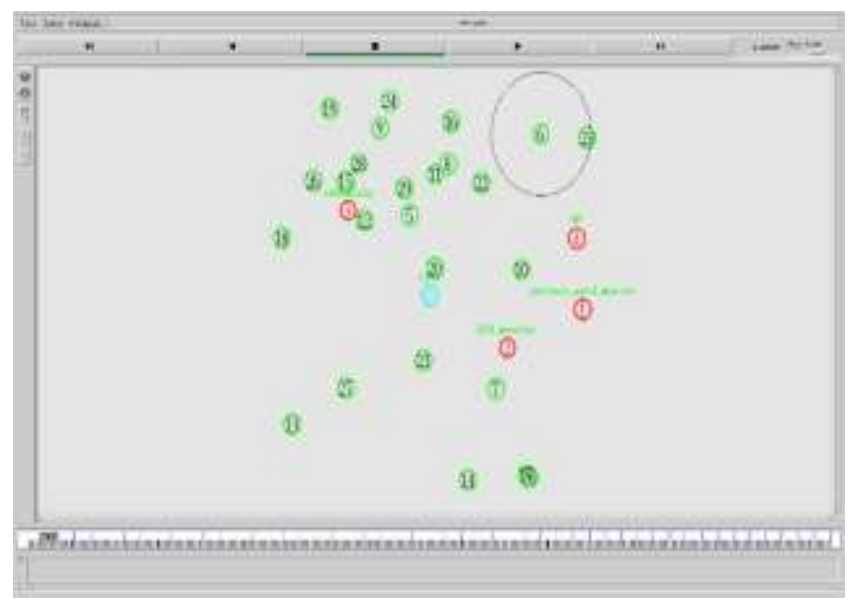

Fig 1. Network Architecture of Healthcare System 


\section{METHODOLOGY}

The FMAC protocol will check whether the data transmitting node is prior node or normal node.

\section{A. Data transfer in normal nodes}

All the sensor nodes in the network transfer the sensed data to the sink node using TDMA protocol. Each node has a time slot to send data. The time is calculated based on packet header length, slot packet length and bandwidth. The normal nodes send their data when the slot time reaches.

slot time $=$

(Header Length/band width) + (slot packet length/band width)

\section{B. Data transfer in prior nodes}

If the sensor node is priority node (i.e. ICU monitor, patient ward monitors etc.), then the prior node send data to sink node using CSMA protocol. In CSMA, the Back-off delay is provided based on packet length and bandwidth. Then the packet transmitted to sink node.

Transmit time $=(8 *$ data length $) /$ band width

\section{Flowchart of FMAC Protocol}

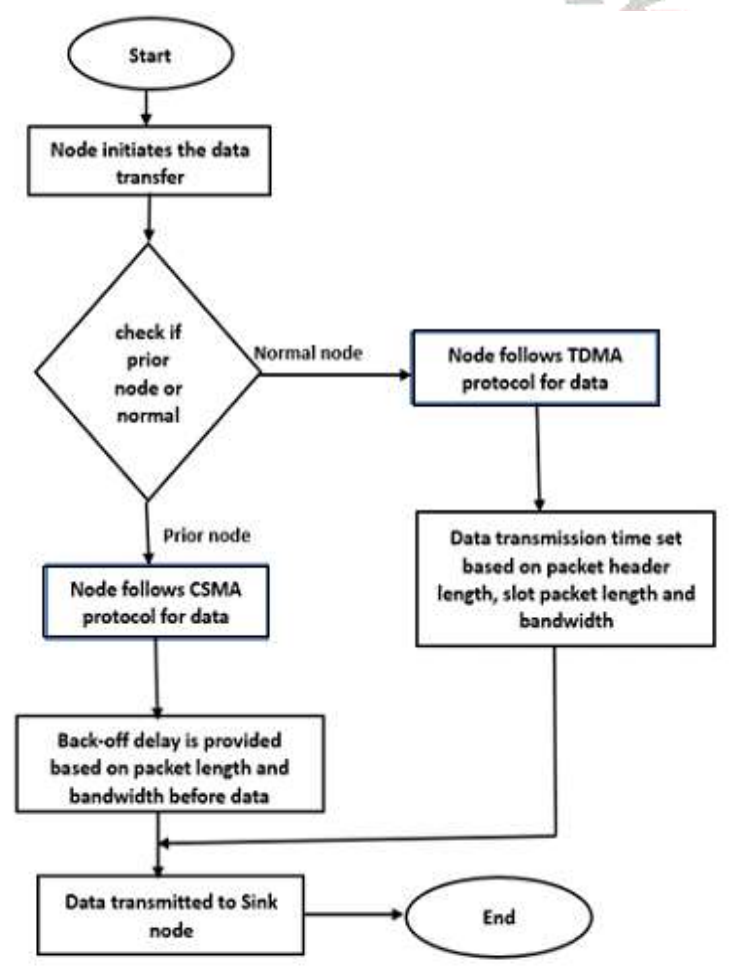

Fig 2. Flowchart For FMAC

\section{D.Implementation Details}

The prior nodes are high priority and they communicate with the center nodes frequently, since it follows CSMA protocol. mac-tdma.cc and mac-tdma.h files have been modified to implement the FMAC protocol. The modification in file mac-tdma.cc is done to check from which node the communication is initiated. If the communicating node is prior node, CSMA MAC protocol will be used for further transmission of data without in delay. But if communicating node is normal node then simple TDMA MAC protocol will be used. Completely generating a new mac layer protocol is not possible, hence we modify the existing TDMA protocol to generate FMAC protocol. The modified protocol is invoked in respective TCL and the modifiers.cc files are added to the ns 2.35 package.

\section{RESULTS}

Our simulation scenario we have deployed 25 common nodes, 4 prior nodes and one sink node. Ad hoc On Demand Distance Vector Protocol (AODV) Routing Protocol is used. Results are taken for varying reporting rate from 10 packets/seconds to 50 packets/seconds. Data transmission between normal nodes and sink node is observed. Data transfer prior nodes also captured.

\section{A. Data transfer in normal nodes}

All the normal nodes send the sensed data using TDMA protocol. Each node has allocated a time slot to sends data. All the sensor nodes in the network transfer the sensed data to the sink node. All the normal nodes send the sensed data using TDMA protocol. In TDMA, it provides different time slots to different transmitters in a cyclically repetitive frame structure. For example, node 1 may use time slot 1 , node 2 time slot 2 , etc. until the last transmitter when it starts over. Each node will send data in allocated time slot.

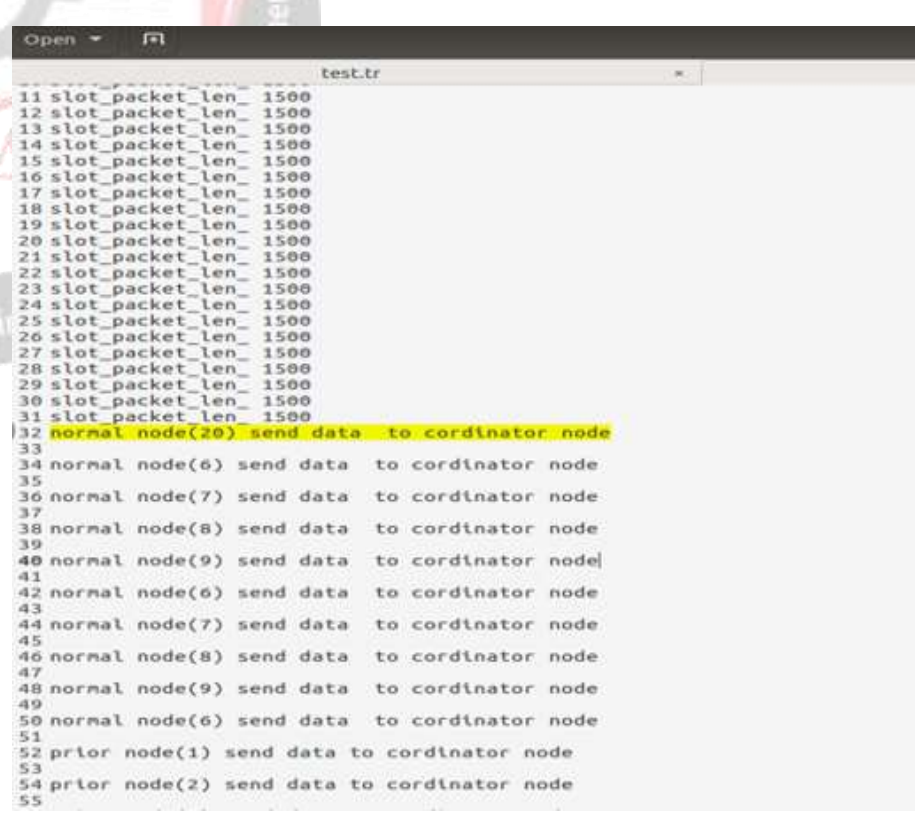

Fig 3. Data transfer between Normal nodes and coordinator node

Fig.3 shows data transfer between normal nodes and coordinator node. In Fig. 4 Nodes like node (20), node (6), node (7), node (8) and node (9) are transferring some data packets to coordinator node. Here these nodes are working in TDMA Media access control protocol where each node send packet to sink in the allotted slot only. Since the node is in one hop distance i.e. the node directly send data to destination without the help of intermediate routers. 


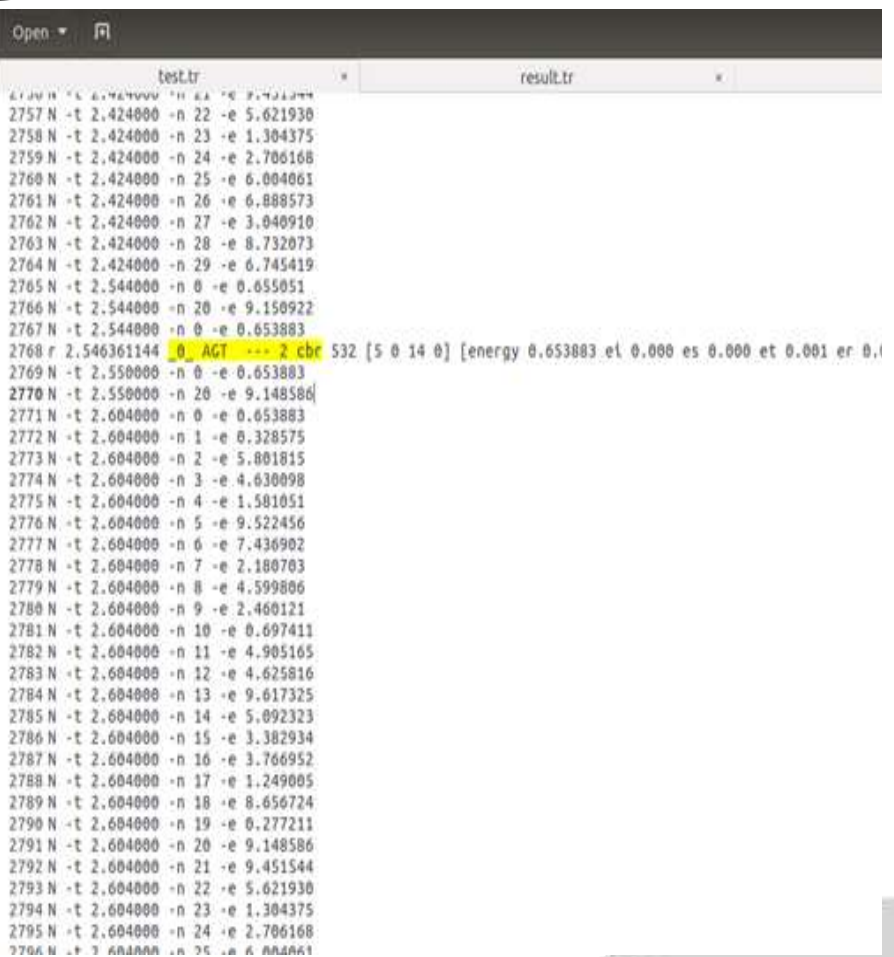

Fig 4. Data Collection at Sink node

Further at sink node data from nodes are collected and processed as shown Fig.4.

\section{B. Data transfer in prior nodes}

In Emergency condition, the nodes send data as prior nodes using CSMA protocol in MAC layer.

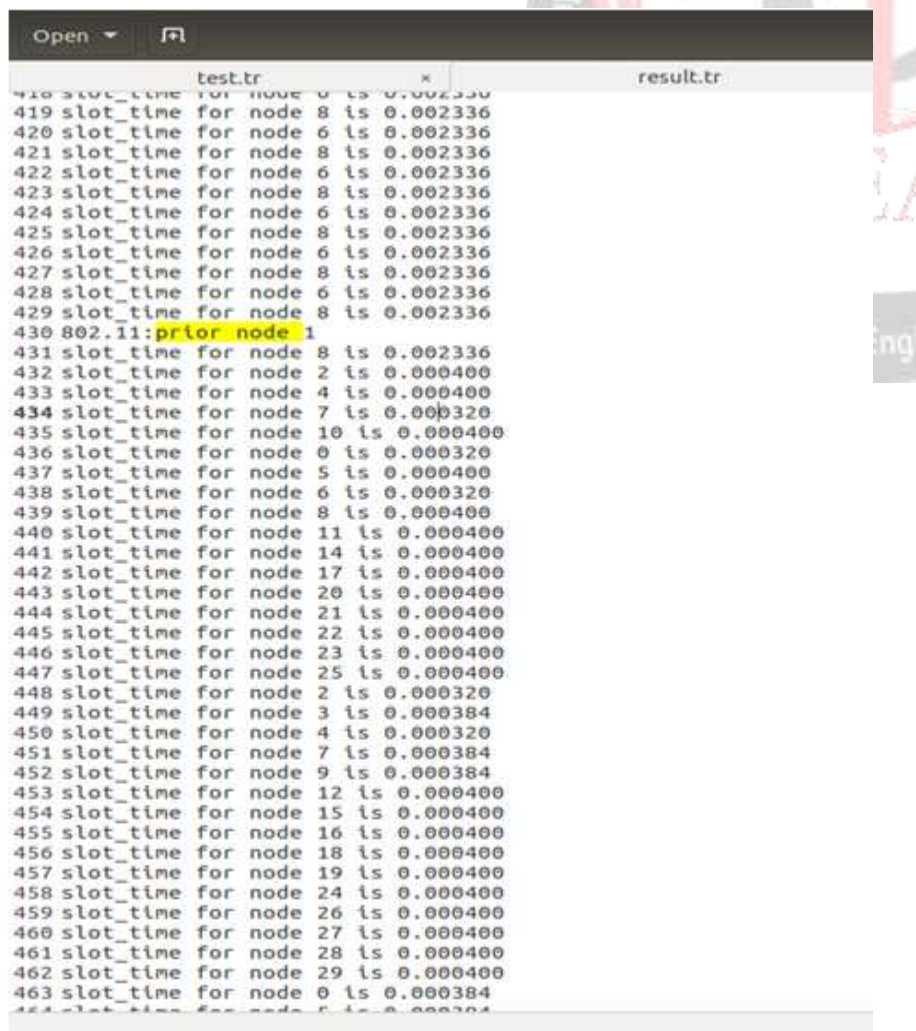

Fig 5. Data transfer in prior nodes

Fig.6 shows the data transfer from prior node (1) so node (1) need not to wait for timeslot as node (1) has high priority it will send data packets using CSMA protocol.

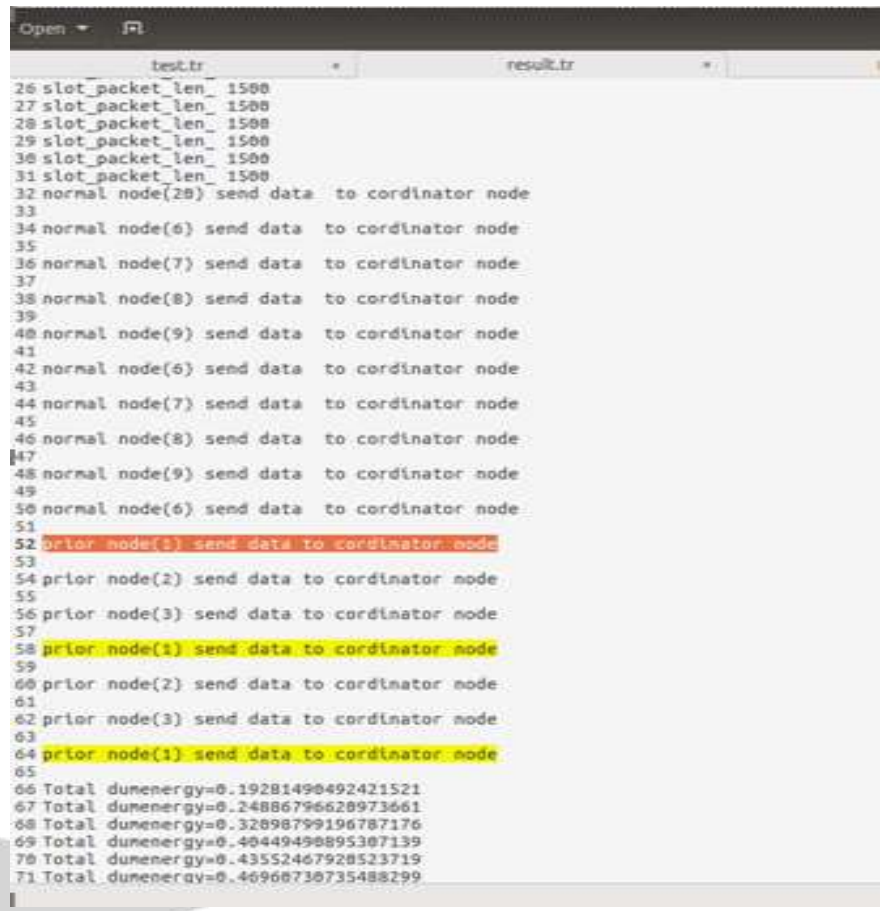

Fig 6. Prior node sends data to coordinator node or sink node

The transmission of data from prior node to coordinator node is shown in Fig 6. Prior node 1,2 and 3 are communicating with coordinator node.

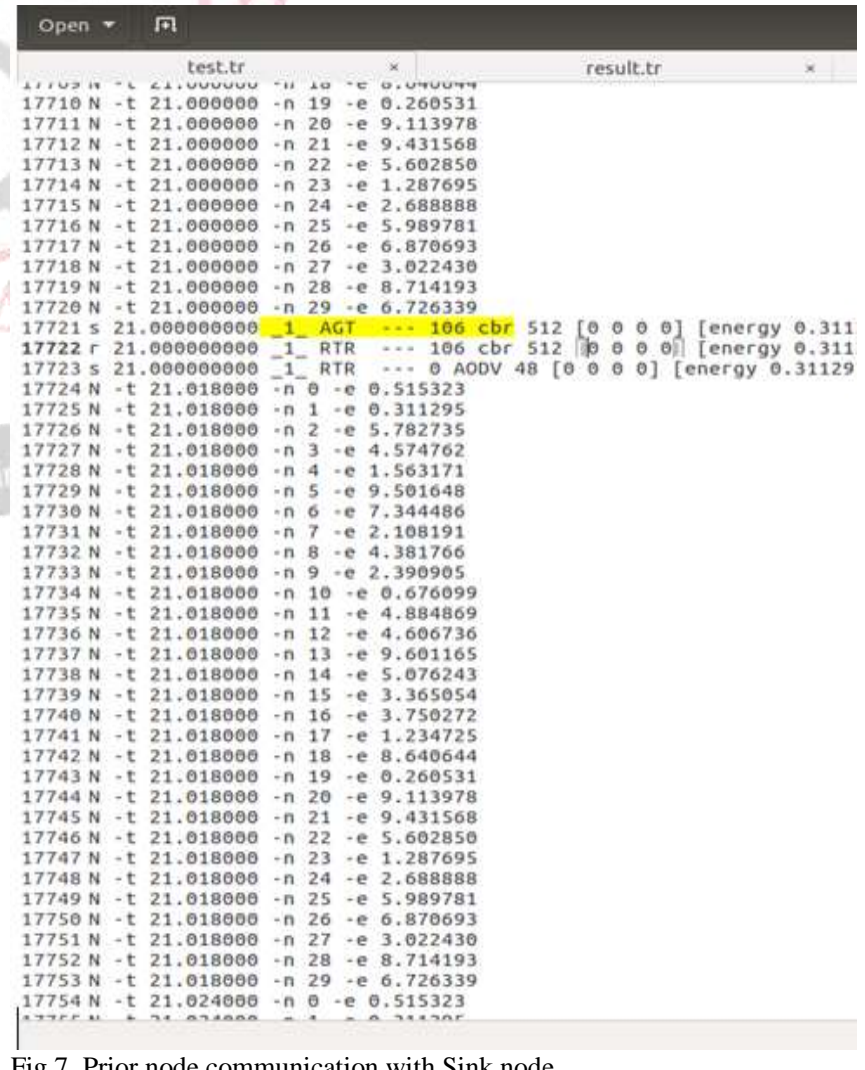

Fig 7. Prior node communication with Sink node

In Fig 7. prior node (1) send data to sink node (0) through path 1-7-0 as sink node is not directly reachable from prior node (1). After arrival of data at Sink node processes it. In the case of prior node data transmission, the node waits for a time named as back-off time to send data. 


\section{CONCLUSION}

In proposed Wireless sensor network implementation of Federated-MAC is done by combining Carrier Sense Multiple Access (CSMA) and Time Division Multiple Access (TDMA) protocols. The performance metric for hospital network are evaluated for varying reporting rate. The implemented protocol found very efficient to tackle emergency situation in Healthcare Monitoring System by applying two different approaches for communication in a network. CSMA is used for communication between prior node and sink node to minimize the delay in communication. Whereas, to improve energy efficiency of each node TDMA Mac protocol is used, which allows each sensor node to remain active in their allotted time slot only. In this Proposed protocol works efficiently for quality of service parameters like Energy Consumption, Packet drop ratio, Packet delivery ratio and throughput irrespective of reporting rate. Here modification in a MAC protocol is done which can improve energy-efficiency to extend network lifetime in wireless sensor networks which is a challenging problem in Healthcare system.

\section{REFERENCES}

[1] Mehmet Yunus Donmmez, Sinan Isik, CemErsoy, " Combined analysis of contention window size and duty cycle for throughput and energy optimization in wireless sensor networks."Vol 57, issue 5,pp.11011112,2012

[2] Liqi Shi, Fapojuwo," TDMA Scheduling with optimized energy efficient and minimum delay in clustered Wireless sensor Networks". IEEE Transaction MC, Vol 9, no 7, pp.227-240, July 2010.

[3] C. Cheng, Member, IEEE, C. K. Tse, Fellow, IEEE, and Francis C.M.L., Senior Member, IEEE," A Delay-Aware Data Collection Network Structure forWireless Sensor Networks", IEEE Sensor journals, Volume. 11, Number. 3 M- 2011.

[4] G. Ekbatanifard, R.Monsefi, M. H. Yaghmaee, S. Hosseini S. "Queen-MAC: A quorum based energy efficient MAC Protocol for Wireless Sensor Networks.", Elsevier, Computer Network, pp.22212236, 2011.

[5] Dattatray S.Waghole, Vivek S. Deshpande "Techniques of Data Collection with Mobile static sinks in Wireless Sensor Networks: A Survey", IJSER,4(10),501-505, 2013.

[6] CSMA based Medium Access Control for Wireless Sensor Network

[7] Dattatray S.Waghole, Vivek S. Deshpande "Performance Analysis of FMAC Protocol for Reporting Rate in Wireless Sensor Networks", Thirteenth International Conference on Wireless and Optical Communications Networks (WOCN),2016

[8] D. S. Waghole, V. S. Deshpande and P. V. Maitri, "Performance analysis of FMAC protocol for packet size in wireless sensor networks," 2015 International
Conference on Pervasive Computing (ICPC), Pune, 2015, pp. 1-5.

[9] Yang, X., Wang, L., Xie, J., \& Zhang, Z., "Energy Efficiency TDMA/CSMA Hybrid Protocol with Power Control for WSN", Wireless Communications And Mobile Computing, 2018, 1-7. doi:10.1155/2018/4168354 